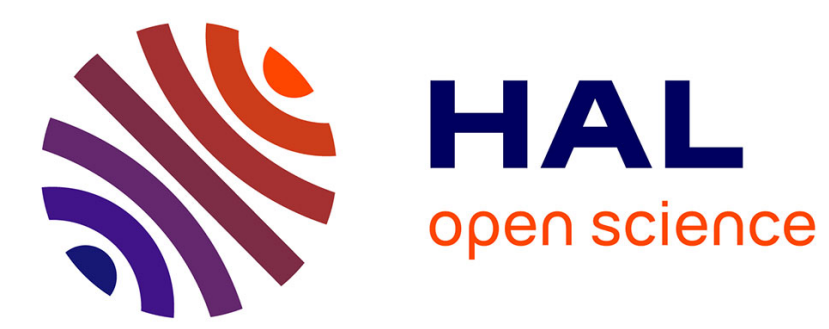

\title{
Référence et existence: une défense de Frege
}

Pascal Ludwig

\section{To cite this version:}

Pascal Ludwig. Référence et existence: une défense de Frege. Quaestio, 2003. ijn_00000500

\section{HAL Id: ijn_00000500 \\ https://hal.science/ijn_00000500}

Submitted on 6 May 2004

HAL is a multi-disciplinary open access archive for the deposit and dissemination of scientific research documents, whether they are published or not. The documents may come from teaching and research institutions in France or abroad, or from public or private research centers.
L'archive ouverte pluridisciplinaire HAL, est destinée au dépôt et à la diffusion de documents scientifiques de niveau recherche, publiés ou non, émanant des établissements d'enseignement et de recherche français ou étrangers, des laboratoires publics ou privés. 


\section{Référence et existence : une défense de Frege}

Gottlob Frege défend, dans de nombreux textes, une conception de l'existence qu'on peut qualifier de déflationniste ${ }^{1}$. Je la reformulerai ainsi pour introduire ma propre discussion :

C'est une erreur de penser que les affirmations ou les dénis d'existence portent sur des objets particuliers. Ils portent sur des concepts et non sur des objets. Lorsque nous disons : «la montagne d'or n'existe pas », nous voulons dire en fait « le concept d'une unique montagne d'or n'est pas instancié ». Et conversement, dire « la montagne d'or existe » revient exactement à dire « le concept d'une unique montagne d'or est instancié (au moins une fois) ».

Cette thèse conduit à nier que l'existence soit une propriété intrinsèque des étants, une propriété qu'ils pourraient ou non instancier. C'est en cela qu'il apparaît légitime de la nommer « déflationniste ». Elle s'oppose à la thèse naïve suivante :

Les affirmations ou les dénis d'existence sont des énoncés qui possèdent une structure logique prédicative. La proposition selon laquelle la montagne d'or existe doit être comprise comme attribuant littéralement la propriété d'exister à la montagne d'or. Conversement, soutenir que la montagne d'or n'existe pas revient à nier que la montagne d'or possède cette propriété.

On considère souvent que la conception déflationniste et la conception naïve sont incompatibles. Cette conviction a conduit de nombreux philosophes à attaquer la position déflationniste de Frege en essayant d'argumenter en faveur de la conception naïve ${ }^{2}$. Je voudrais montrer dans cet article qu'il s'agit là d'une erreur. Ma stratégie sera la suivante. Je commencerai par distinguer deux thèses frégéennes, l'une ayant une portée métaphysique, l'autre une portée sémantique. Je défendrai ensuite la thèse métaphysique contre quelques arguments apparemment décisifs qu'on pourrait lui

${ }^{1}$ Voir en particulier G. FREge, « Dialogue avec Pünjer sur l'existence », trad. fr. ALI BenMACKhlouf, in P. DE ROUILLHAN / C. TIERCELIN (éd. par), Gottlob Frege : Écrits posthumes, Jacqueline Chambon, Nîmes, 1994, 67-84. Voir également G. FREGE, Les fondements de l'arithmétique, trad. fr. C. IMBERT, Seuil, Paris, 1969.

2 Pour une tentative particulièrement aboutie, voir G. Evans, The Varieties of Reference, Oxford University Press, Oxford, 1982, chap. 10. 
opposer. Enfin, je soutiendrai que la thèse sémantique est en revanche vraisemblablement fausse. On peut ainsi concilier une conception naïve de l'existence en sémantique avec une conception frégéenne, déflationniste, en métaphysique. Ce qui revient à dire que la théorie frégéenne est, pour l'essentiel, parfaitement correcte.

1.

L'hypothèse déflationniste possède une dimension révisionniste, ou réformiste. Elle repose sur une dénonciation des errements dans lesquels peuvent nous conduire les «mers du langage » : notre pratique linguistique nous pousse à négliger une analyse simple et élégante de certaines classes d'énoncés, pour privilégier une analyse paradoxale, sinon simplement absurde.

L'analyse de Frege s'enracine dans ses réflexions sur les concepts numériques, comme « avoir trois satellites », ou « avoir 400 feuilles ». Si l'on se fie à la forme grammaticale des énoncés où figurent de tels concepts, ceux-ci semblent attribuer des propriétés à des objets. Il existe en effet un parallélisme frappant entre (1) et (2) d'une part, (3) et (4) de l'autre :

(1) Ce livre a 350 pages.

(2) Ce livre a une couverture en cuir.

(3) Les feuilles de l'arbre sont 200.

(4) Les feuilles de l'arbre sont vertes.

De cette ressemblance grammaticale, on pourrait tirer l'idée selon laquelle les concepts numériques, comme « être 200 », expriment des propriétés, et en conséquence identifier les nombres à des propriétés des choses.

Il s'agit, pour Frege, d'une illusion. Il présente plusieurs arguments contre l'identification des nombres à des propriétés, dont le plus convaincant est le suivant ${ }^{3}$ :

Une entité ne possède pas de nombre en elle-même, de façon absolue, mais uniquement relativement à un concept sortal que l'on utilise pour la qualifier. Le nombre n'est donc pas une propriété des objets, comme la couleur, mais une propriété de certains concepts, les concepts sortaux, qui permettent de qualifier les objets.

\footnotetext{
${ }^{3}$ Il ne s'agit pas d'une citation mais d'une reconstruction de l'argument. Voir FREGE, Fondements, cit., 175-176.
} 
Ainsi, le livre ne possède pas le nombre « 350 » en soi, ou absolument ; il possède 350 pages : c'est l'application du concept sortal de page au livre qui permet de lui appliquer un nombre. Mais l'application d'un concept sortal différent peut déboucher sur un nombre différent : le livre a 350 pages, mais peut-être n'a-t-il que 12 chapitres, 2 parties, et une unique table des matières.

Frege conclut que les concepts numériques expriment des propriétés de concepts sortaux, ou, comme il le dira plus tard, des concepts de second ordre. De façon intuitive, on peut appliquer le concept numérique $N$ à un concept sortal si, et seulement si, l'extension de ce concept possède un cardinal égal à $N$. La définition n'est qu'en apparence circulaire, pour des raisons sur lesquelles je ne m'attarderai pas ici, mais qui apparaissent clairement si l'on considère le cas particulier du nombre zéro :

«Le nombre 0 appartient à un concept si, quel que soit $a$, il est toujours vrai que $a$ ne tombe pas sous ce concept $»^{4}$.

On voit qu'il n'est pas nécessaire de présupposer de connaissance préalable de la signification des concepts numériques pour pouvoir définir le prédicat de second ordre « 0 appartient à » : ce prédicat peut être correctement appliqué à un concept quelconque s'il possède une extension vide, c'est-à-dire s'il n'est pas instancié.

Dans les Fondements de l'arithmétique, Frege remarque en passant que les attributions d'existence peuvent être analysées de façon semblable aux attributions de nombres :

«La proposition qu'il n'y a pas de triangle rectangle rectiligne équilatéral énonce une propriété du concept 'triangle rectangle, rectiligne, équilatéral' ; elle lui attribue le nombre zéro. À cet égard, l'existence a quelque analogie avec le nombre. Affirmer l'existence, ce n'est rien d'autre que nier le nombre zéro $»^{5}$.

L'analogie, faudrait-il ajouter, n'est pas absolument parfaite. On ne peut attribuer de nombres qu'aux concepts sortaux, mais pas aux termes de masse. En revanche, on peut tout à fait nier l'existence d'une substance désignée par un terme de masse. Par exemple :

(5) L'or violet n'existe pas.

qui selon Frege s'analyse ainsi :

(6) Le concept « or violet » n'est pas instancié.

\footnotetext{
${ }^{4}$ FrEGE, Fondements, cit., 183.

${ }^{5}$ FREGE, Fondements, cit., 180.
} 
Frege rapproche aussi les concepts numériques des quantificateurs, et l'on peut donc procéder à un rapprochement similaire entre les quantificateurs et les attributions d'existence ${ }^{6}$. Des énoncés quantifiés, comme :

(7) Au moins un homme marche dans le parc.

(8) Toutes les baleines sont des mammifères.

ne portent qu'en apparence sur des objets particuliers. C'est surtout évident pour (8) : s'il s'agissait d'une attribution de propriété à un objet, ou, de façon plus plausible, à une collection d'objets (une collection de baleines), l'énoncé ne pourrait pas être vrai s'il n'existait pas de baleines. Or, (8) demeurerait vrai en l'absence de baleine. L'énoncé ne porte donc pas sur des baleines particulières, mais sur des concepts : il affirme qu'il existe une relation de subordination entre le concept « baleine » et le concept « mammifère ».

Résumons le point essentiel de notre discussion : le verbe exister n'exprime jamais, selon Frege, une propriété ; il faut l'analyser de façon syncatégorématique, à partir du contexte dans lequel il apparaît. On se rendra alors compte qu'affirmer l'existence, c'est toujours affirmer qu'il y a certains objets tombant sous un concept.

\section{2.}

Examinons maintenant deux arguments frégéens importants justifiant l'approche déflationniste ${ }^{7}$.

Le premier repose sur deux principes, que nous formulerons ainsi :

1 Principe de négation signifiante : l'attribution d'une propriété à un objet doit véhiculer un contenu d'information ; le fait qu'une propriété soit attribuée à un objet implique donc que certains objets ne possèdent pas la propriété.

2 Principe de l'existence des objets de référence : quelle que soit l'analyse qu'on donne du verbe « exister », tout objet auquel on peut faire référence dans un jugement existe.

Une fois acceptés ces principes, le raisonnement suivant peut être reconstruit :

1 Si l'existence est une propriété, on l'attribue à l'aide d'énoncés singuliers ayant pour forme logique « $E a »$.

2 Dans «Ea», « $a »$ fait référence à un objet.

${ }^{6}$ FREGE, Fondements, cit., 176-177.

${ }^{7}$ La reconstruction qui suit reprend pour l'essentiel l'argument de FREGE, « Dialogue avec Pünjer sur l'existence », cit, 67-84. 
3 Donc, $a$ existe, en vertu du principe de l'existence des objets de référence.

4 Mais alors, $a$ ne peut pas manquer de posséder la propriété attribuée par le prédicat $« E »$. Le principe de négation signifiante est donc violé.

5 En conséquence, l'existence n'est pas une propriété.

Il s'agit d'un argument valide logiquement, et la seule façon d'éviter sa conclusion est de rejeter l'un des deux grands principes formulés par Frege.

Le premier principe est fort bien justifié par Frege, comme l'a montré Stéphane Chauvier dans sa contribution au présent volume, et je propose de l'accepter sans plus de discussion.

Le second apparaît en revanche discutable, et l'on pourrait penser de prime abord que l'accepter revient à commettre une véritable pétition de principe. Pourquoi ne pourraiton pas faire référence à des objets qui n'existent pas ? On peut trouver, sinon une réponse, du moins les prémisses d'une réponse à cette interrogation dans l'oeuvre de Frege.

On sait en effet que, pour Frege, la notion d'objet est seconde par rapport à celle de référence. Un objet n'est rien d'autre pour lui qu'une entité à laquelle on peut faire référence, à l'aide d'un nom propre apparaissant dans une phrase susceptible de vérité ou de fausseté. Voici la façon dont Michael Dummett décrit cette position :

«L'usage par Frege du terme ontologique 'objet' est strictement corrélatif de son usage du terme linguistique 'nom propre' : tout ce dont un nom propre peut tenir lieu est un objet, et parler de quelque chose comme d'un objet revient à affirmer qu'il y a, ou qu'au moins il pourrait y avoir, un nom propre en tenant lieu $»^{8}$.

Si l'on accepte la thèse selon laquelle un objet n'est rien d'autre que ce qui peut être nommé par un nom propre, on peut établir de façon convaincante que tout objet existe, ou, pour le dire autrement, que tout ce à quoi l'on peut faire référence existe. Frege considère en effet que l'inférence nommée généralisation existentielle, qui permet de conclure de l'attribution d'une propriété à un objet à l'affirmation selon laquelle au moins un objet possède cette propriété, est une loi logique fondamentale :

$\mathrm{Fa}$

$\exists x F x$

Selon cette loi, le fait d'attribuer la propriété $F$ véridiquement à un objet désigné par un nom propre « $a »$ implique qu'il existe au moins un objet possédant la propriété. Or,

8 M. Dummett, Frege. Philosophy of Language, Duckworth, London, 1973, 55. 
cette règle inférentielle est invalidée si l'on accepte que certains objets pouvant donner lieu à un acte de référence n'existent pas. Cette discussion nous permet de reformuler l'argument de Frege de la façon suivante :

1. Pour attribuer une propriété à quoi que ce soit, il faut y faire référence.

2. On ne peut faire référence qu'à des objets existants.

3. Les énoncés d'existence n'attribuent pas de propriété.

La conclusion 3 suit de 1 et de 2 si l'on accepte le principe de négation signifiante. Il y a en effet deux types possibles d'énoncés d'existence : les énoncés qui affirment l'existence, et ceux qui la dénient. La possibilité des premiers est exclue, si l'existence est une propriété, puisque l'acte de référence lui-même présuppose l'existence. La possibilité des seconds entraînerait, quant à elle, des absurdités logiques. Considérons en effet la formalisation en logique du premier ordre d'un déni d'existence :

(9) Pégase n'existe pas.

(10) $\neg E p$

La loi de généralisation existentielle nous permet d'affirmer que (11) est une conséquence logique de (10) :

(11) $\exists x \neg E x$

Ce qui se lit : il existe au moins un $x$ tel que $x$ n'existe pas. Il s'agit, pour un frégéen, d'une violation évidente du principe de contradiction. Comme le remarquera G. E. Moore :

«Il semble (...) que dire de quelque chose que ce soit que nous pouvons mentionner qu'elle n'est absolument pas revienne à se contredire : de sorte que toute chose que nous pouvons mentionner doive posséder quelque sorte d'être $»^{9}$.

Or, s'il est absurde de dénier l'existence d'un objet, il est conversement tautologique de l'affirmer d'un objet. L'existence n'est donc pas une propriété, et « exister » n'est pas une expression prédicative.

Il est facile de négliger la radicalité de la conclusion de Frege. Celui-ci ne nie pas que certains types d'objets - par exemple, les objets simplement possibles, comme l'unique fils de Kant, ou les objets impossibles comme le cercle carré - existent. De façon beaucoup plus profonde, il remet en question le sens même du discours sur l'existence des objets. Selon lui, lorsque nous nous interrogeons sur l'existence effective du fils de Kant, nous ne nous interrogeons pas vraiment sur un objet, mais bel et bien

${ }^{9}$ G. E. Moore, Some Main Problems in Philosophy, George Allen and Unwin, London, 1953, 289. 
sur un concept, celui de « fills de Kant», dont nous nous demandons s'il est ou non instancié. S'interroger sur le mode d'être, ou le type d'être d'un objet, n'a donc selon Frege rigoureusement aucun sens ${ }^{10}$.

\section{3.}

La conception déflationniste de l'existence a eu un immense succès jusqu'au début des années 1960 en philosophie analytique. Elle permet d'établir une relation élégante entre logique et métaphysique, et donc, si l'on considère que les thèses scientifiques les mieux établies peuvent être formulées dans un vocabulaire logique, entre science et métaphysique. Ce que l'on peut nommer d'une façon douée de sens, c'est ce qu'il y a dans le domaine de quantification; et ce qu'il y a dans ce domaine, ce n'est rien d'autre que l'ensemble des choses susceptibles d'être la valeur des variables liées présentes dans les formules exprimant nos thèses scientifiques les mieux justifiées. Mais le principe central d'où découle cette conception est-il vraiment acceptable ? Doit-on identifier un étant, un objet existant, à quelque chose que l'on peut nommer ?

Le développement de la logique modale quantificationnelle, et la formulation d'une sémantique pour cette logique par Jaakko Hintikka et Saul Kripke, ont conduit de nombreux philosophes à reconsidérer cette question. Il semble en effet que l'on puisse facilement former des contre-exemples à la thèse métaphysique de Frege dès lors que l'on se place dans un langage comprenant des modalités. Considérons en effet les énoncés suivants :

(12) J'existe, mais j'aurais pu ne pas exister.

${ }^{10}$ Cette conclusion sera amplifiée et dramatisée par Ludwig Wittgenstein, dans le Tractatus-logico philosophicus. Un des thèmes majeurs de cet ouvrage réside dans le développement de l'idée frégéenne selon laquelle l'existence individuelle n'est en aucun cas une propriété attribuable à des objets de façon douée de sens. On peut, selon le Tractatus, décrire un objet en disant comment il est, mais certainement pas dire ce qu'il est, le type d'être qu'il se trouve avoir : « une proposition peut dire comment est une chose, non ce qu'elle est $\gg$ (L. WiTTGENSTEIN, Tractatus-logico philosophicus, trad. fr. G.-G. GRANGER, Gallimard, Paris, 1993, 43). Nous manifestons nos engagements ontologiques en employant dans notre langage des noms propres permettant de faire référence à des objets et de décrire leurs propriétés. Mais nous ne pouvons pas discourir sur ces engagements métaphysiques : " la logique remplit le monde ; les frontières du monde sont aussi ses frontières. Nous ne pouvons pas dire en logique : il y a ceci et ceci dans le monde, mais pas cela » (L. WiTTGENSTEIN, Tractatus, cit., 93). 
(13) Pégase ${ }^{11}$ n'existe pas, mais Pégase aurait pu exister.

Dans (12), je fais référence à une personne existant dans le monde réel, tout en affirmant qu'elle n'existe pas dans un monde possible au moins. Dans (13), il semble que je fasse référence à un objet qui, quoique n'existant pas actuellement, dans le monde réel, aurait pu y exister, c'est-à-dire existe dans un monde possible. Dans les deux cas, une indépendance semble s'instaurer entre référence et existence.

L'hypothèse d'une telle indépendance est-elle bien fondée ? Pour répondre à cette question, nous nous proposons d'examiner la sémantique des concepts modaux, telle qu'elle est formalisée dans les systèmes de logique modale quantificationnelle. Un tel point de départ nous semble pertinent, puisque la logique des modalités constitue certainement le meilleur accès à la signification des concepts de possibilité et de nécessité.

L'idée intuitive sur laquelle repose la sémantique des langages modaux quantificationnnels est simple : la définition récursive de la vérité pour les formules appartenant à de tels langages est relativisée non seulement à un modèle, mais aussi à un monde possible. Une formule vraie, dans un modèle, relativement à un monde possible, peut être fausse, dans ce même modèle, relativement à un autre monde possible. D'un point de vue logique, l'expression « monde possible » désigne l'élément d'un ensemble, nommé « ensemble de mondes », relativement auquel une formule interprétée peut être évaluée comme vraie ou comme fausse. Lorsque le logicien modal quantifie sur des « mondes possibles », il n'est donc engagé ontologiquement qu'à reconnaître l'existence de certains types d'ensembles. Il est essentiel de distinguer l'idiome des mondes possibles employé en logique modale de celui employé en métaphysique : un philosophe peut reconnaître l'utilité de la sémantique modale, et donc quantifier sur des ensembles de mondes, sans pour autant penser qu'il existe d'autres mondes que le monde réel. Lorsque nous dirons qu'il existe un monde possible relativement auquel une proposition, fausse dans le monde réel, est vraie, nous voudrons simplement dire

${ }^{11}$ Pour les besoins de l'argument, je supposerai dans cet article que l'on peut nommer les objets simplement possibles. La discussion dont cette hypothèse fait l'objet - voir en particulier S. KRIPKE, Naming and Necessity, Cambridge, Mass., Harvard University Press, 1990, Postface - me semble en effet indépendante des principales thèses défendues ici. 
que le monde réel aurait pu être tel que cette proposition fût vraie, sans nous engager quant à l'existence réelle d'autres mondes que le monde actuel12.

Alors qu'un modèle pour la logique du premier ordre comprend un domaine de discours, qui est l'ensemble des particuliers pouvant servir de valeurs aux variables liées par des quantificateurs, et une fonction d'interprétation assignant des extensions aux différents prédicats du langage, un modèle pour la logique modale quantificationnelle comprend aussi un ensemble de mondes possibles, et une relation $R$ définie sur cet ensemble, nommée relation d'accessibilité. L'utilisation de telles structures ensemblistes - les ensembles de mondes, ajoutés aux ensembles d'individus - permet d'analyser la vérité de formules comportant des opérateurs modaux dans un métalangage qui n'est pas modal, c'est-à-dire un métalangage n'utilisant d'aucune façon les concepts de nécessité et de possibilité. L'analyse des deux modalités est la suivante : « Nécessairement $\phi$ » est vraie dans un modèle $M$ et dans un monde possible $\omega$ si et seulement si « $\phi »$ est vraie dans ce modèle relativement à tous les mondes possibles $\omega^{\prime}$ accessibles à partir de $\omega$. «Possiblement $\phi$ » est vraie dans un modèle $M$ et dans un monde possible $\omega$ si et seulement si « $\phi$ » est vraie dans ce modèle relativement à $a u$ moins un monde possible $\omega^{\prime}$ accessible à partir de $\omega$. On peut montrer que certaines formules modales permettent de caractériser les propriétés de la relation d'accessibilité. Si l'on veut donner un sens métaphysique aux modalités, il est bon de considérer que la relation d'accessibilité est une relation d'équivalence, c'est-à-dire une relation transitive, symétrique et réflexive. La relation doit être réflexive : un monde possible donné est certainement accessible à partir de lui-même, puisqu'une proposition vraie doit être possiblement vraie. D'autre part, la relation doit être transitive, si l'on veut qu'une proposition nécessairement vraie soit aussi nécessairement nécessairement vraie. Enfin, elle doit être symétrique. Dire d'un monde qu'il est possible au sens métaphysique ne doit dépendre en rien de notre pouvoir de conceptualisation ou de nos connaissances ; il serait donc absurde qu'un monde soit possible relativement au monde réel, mais que le monde réel ne soit pas possible relativement à ce monde. On nomme S5 le système modal dans lequel la relation d'accessibilité possède ces trois caractéristiques. C'est sur ce système particulier que nous ferons porter nos analyses,

12 Je soutiendrai dans la suite de l'article qu'un engagement ontologique vis-à-vis d'entités simplement possibles est justifié. Il me semble cependant que l'existence de telles entités n'implique pas celle de mondes possibles, pas plus que l'existence des nombres n'implique celle d'un monde des nombres. 
car, comme on l'a souvent remarqué, c'est celui qui possède le plus de pertinence lorsqu'on s'intéresse au sens métaphysique des modalités (par opposition, par exemple, à leur sens déontique ou épistémique) ${ }^{13}$.

Dans la sémantique la plus simple pour un langage modal quantificationnel, il existe un unique domaine de quantification commun à tous les mondes possibles de la structure ensembliste utilisée pour interpréter les formules. Si la relation d'accessibilité est une relation d'équivalence, comme nous l'avons stipulé, et si le domaine de quantification est le même pour tous les mondes possibles, les formules $\mathbf{B F}$ et $\mathbf{C B F}$, dont Ruth Barcan Marcus a été la première à déceler l'importance ${ }^{14}$, sont des vérités logiques (elles sont vraies dans tous les modèles et pour tous les mondes possibles de ces modèles) :

BF : $\forall x \square \phi x \rightarrow \square \forall x \phi x^{15}$

Ce qu'on peut paraphraser intuitivement ainsi : si toute chose possède nécessairement une certaine propriété $\phi$, alors nécessairement, toute chose possède cette propriété $\phi$. Il est plus facile de saisir les conséquences métaphysiques de cette formule si l'on considère la formule logiquement équivalente suivante :

$$
\text { BF' : } \diamond \exists x \phi x \rightarrow \exists x \diamond \phi x
$$

Cette formule, intuitivement, représente la proposition suivante : s'il avait pu être le cas qu'il y ait une chose possédant la propriété $\phi$, alors il y a une chose dont il aurait pu être le cas qu'elle ait la propriété $\phi$. Les hypothèses sémantiques que nous avons présentées impliquent que la formule converse de Barcan, qui est la formule suivante, appartient également à l'ensemble des théorèmes de notre théorie des modalités :

CBF : $\square \forall x \phi x \rightarrow \forall x \square \phi x$

De nouveau, la formulation logiquement équivalente suivante est plus éclairante pour l'intuition :

CBF' $\exists x \oslash \varphi x \rightarrow \diamond \exists x \varphi x$

\footnotetext{
${ }^{13}$ Hughes et Cresswell notent ainsi que « S5 reflète (...) un sens 'absolu' du mot 'concevable' - un sens dans lequel dire d'un état de chose qu'il est concevable revient à dire quelque chose à son sujet, sans aucune référence aux capacités de conceptualisation qui existent ou non dans d'autres états de choses ». Voir G. E. Hughes / M. J. CRESSWELL, An Introduction to Modal Logic, Routledge, London, 1968, 79.

14 Voir les articles réunis dans R. BARCAN MARCus, Modalities, Oxford University Press, Oxford,1993.

15 « $\square$ » est l'opérateur de nécessité, et se lit « nécessairement »; « $\diamond »$ est l'opérateur de possibilité, et se lit «possiblement».
} 
On peut la paraphraser ainsi : s'il y a quelque chose qui aurait pu avoir la propriété $\phi$, alors il aurait pu être le cas que quelque chose ait la propriété $\phi$.

Les formules BF et $\mathbf{C B F}$ ont une importante propriété : elles peuvent être déduites formellement du système d'axiomes le plus simple pour la logique modale quantificationnelle ${ }^{16}$. On pourrait donc penser qu'il s'agit de lois logiques fondamentales de la logique modale, ayant un véritable statut régulateur en ce qui concerne la métaphysique des modalités.

Pourtant, certains auteurs ont avancé des contre-exemples qui paraissent de prime abord décisifs aux formules de Barcan ${ }^{17}$. Considérons les énoncés suivants, dans lesquels nous supposerons que Pierre Martin n'a, dans le monde réel, pas eu de fils :

(14) Pierre Martin aurait pu avoir un fils.

(15) Il existe une personne qui aurait pu être le fils de Pierre Martin.

On peut représenter ces deux énoncés ainsi18 :

(16) $\diamond \exists x F x a$

(17) $\exists x \diamond F x a$

Dans un système dans lequel BF est un théorème, (16) implique logiquement (17).

Or, l'argument correspondant en langue naturelle ne semble pas valide : n'est-il pas en effet évident qu'il puisse exister un fils de Pierre Martin dans un monde possible, sans pour autant qu'il existe dans le monde actuel une personne réelle qui aurait pu être son fils? Cette conclusion semble manifestement fausse, puisqu'on peut raisonnablement soutenir qu'aucune des personnes réellement existantes dans le monde n'aurait pu être le fils de Pierre Martin.

D'un point de vue sémantique, c'est parce que les domaines de tous les mondes possibles postulés dans la théorie doivent posséder exactement les mêmes individus

${ }^{16}$ B. Linsky et E. Zalta insistent fortement, et à juste titre, sur le fait que les systèmes modaux quantificationnels les plus simples possèdent $\mathbf{B F}$ et $\mathbf{C B F}$ dans l'ensemble de leurs théorèmes. Voir B. LinsKY / E. ZALTA, «In defense of the simplest quantified modal logic », Philosophical Perspectives, 8 (1994), 431-458.

17 Dans toute la discussion sur les contre-exemples à $\mathbf{B F}$ et $\mathbf{C B F}$, je m'appuie sur les publications suivantes : Hughes / CRESSwELl, An Introduction to Modal Logic, cit., 170-190 ; T. PARSONS, « Ruth Barcan Marcus and the Barcan Formula », in W. SinnotT-Armstrong, (ed.), Modality, Morality and Belief : Essays in Honor of Ruth Barcan Marcus, Cambridge University Press, Cambridge, 1995, 3-12 ; T. WILLIAMSON, « Bare possibilia », Erkenntnis, 48 (1998), 257-273.

18 Où $« a »$ est une constante d'individu, qui dans l'interprétation visée désigne Pierre Martin, et $« F$ » est une lettre de prédicat binaire qui dénote l'ensemble des couples $\langle x, y\rangle$ tels que $x$ est le fils de $y$. 
comme éléments que la forme inférentielle fautive est valide. Il semble intuitivement qu'il puisse « exister », dans un monde possible, plus d'individus qu'il n'en existe dans le monde réel - ce qui revient à soutenir que d'autres objets que ceux qui existent effectivement auraient fort bien pu exister. En toute généralité, l'argument suivant n'apparaît pas logiquement correct19 :

A : Cela aurait pu être le cas qu'il y ait quelque chose qui diffère de toutes les choses qui existent dans le monde actuel. En conséquence, il existe dans le monde actuel quelque chose qui aurait pu différer de toutes les choses existant actuellement.

La prémisse semble vraie : il paraît intuitivement convaincant de soutenir que certains objets, qui n'existent pas actuellement, auraient pu néanmoins exister dans d'autres mondes possibles que le monde actuel. Mais la conclusion paraît fausse : chaque chose existant dans le monde actuel est nécessairement identique à elle-même, et il n'est donc pas possible que l'une de ces choses diffère de toutes les choses existant dans le monde actuel. Cela impliquerait qu'elle diffère d'elle-même, au moins dans un monde possible. Pourtant, A possède une forme logique valide, si l'on suppose que BF est une vérité logique.

Des contre-exemples à $\mathbf{C B F}$ ont également été proposés ${ }^{20}$. Supposons qu'il y ait strictement moins d'objets dans un monde possible donné que dans le monde réel. Cela semble tout à fait concevable : j'aurais pu, par exemple, ne pas exister, puisque mon existence est contingente. En conséquence, il existe un monde possible dans lequel aucune personne n'est identique à Pascal Ludwig. (18) est donc vrai :

(18) Il y a une personne telle qu'il eût été possible qu'aucune personne ne lui fût identique.

En revanche, le fait qu'une personne soit identique à elle-même est une nécessité métaphysique. Il n'est donc pas possible qu'il existe une personne telle qu'aucune personne ne lui soit identique : il y en aura toujours une, elle-même, avec laquelle elle sera identique. (19) est donc faux :

(19) Il eût été possible qu'il y ait une personne telle qu'aucune personne ne soit identique avec elle.

19 Pour cette discussion, voir particulièrement PARSONS, « Ruth Barcan Marcus and the Barcan Formula », cit., 7-11.

20 J'emprunte la présentation du contre-exemple qui suit à WILLIAMSON, « Bare possibilia », cit., 258. 
Pourtant, l'argument suivant est valide si l'on accepte CBF :

Il existe (réellement) une entité telle qu'il aurait pu n'y avoir rien d'identique à

elle. En conséquence, il aurait pu exister une entité telle que rien (pas même ellemême) ne lui soit identique.

Puisque la conclusion de cet argument est manifestement absurde, accepter CBF contraint à rejeter la prémisse de l'argument, ce qui semble contredire nos intuitions modales.

Ces contre-exemples aux formules de Barcan apparaissent tous motivés par une unique intuition métaphysique fondamentale. Derrière eux se profile la thèse selon laquelle il y a, dans certains mondes possibles, des objets qu'il n'y a pas dans le monde réel ; ou, inversement, qu'il peut y avoir dans le monde réel des objets qui, dans certains mondes possibles, n'existent pas. Cette intuition semble contredire le premier principe de Frege: il paraît possible de faire référence à des objets qui n'existent pas, et il semble que cette pratique soit courante dans nos raisonnements modaux. Puisqu'un objet auquel on peut faire référence peut être la valeur d'une variable de quantification, cela implique qu'on peut quantifier sur des objets qui n'existent pas.

La sémantique défendue par Saul Kripke cherche à traduire cette intuition métaphysique ${ }^{21}$. Dans cette sémantique, l'hypothèse d'un unique domaine de quantification, commun à tous les mondes possibles, est abandonnée. Intuitivement, il peut y avoir dans les mondes possibles des objets qu'il n'y a pas dans le monde réel, et inversement, il y a (éventuellement) dans le monde réel des objets qu'il n'y a pas dans les autres mondes possibles.

Il est aisé, dans ce nouveau cadre sémantique, de construire des contre-modèles aux formules de Barcan, censés correspondre aux contre-exemples que nous venons d'analyser. Les formules de Barcan, nous l'avons vu, peuvent être dérivées de l'axiomatisation la plus simple de la logique modale quantificationnelle, fondée sur le système propositionnel modal $S 5$. Quel axiome faut-il donc abandonner pour que l'axiomatisation soit fiable dans cadre de l'hypothèse kripkéenne des domaines variables ? Considérons le schéma d'axiome d'instanciation universelle, qui comporte toutes les formules de la forme :

21 Voir S. KRIPKE, « Semantical considerations on modal logic », Acta Philosophica Fennica,16 (1963), 83-94. 
IU $\forall x \phi \rightarrow[y / x] \phi$, où $y$ est libre pour $x$ dans $\phi$, et où $[y / x] \phi$ est le résultat du remplacement de toutes les occurrences libres de $x$ par $y$ dans $\phi$.

Les formules de cette forme ne peuvent être satisfaites dans tous les modèles de Kripke si l'on accepte des domaines variables, précisément parce qu'il peut, dans un monde possible, y avoir un objet de référence qui n'appartient pour autant pas au domaine de quantification du monde en question. Supposons que l'on évalue une formule du type IU, disons (20) dans un monde $\omega$ :

(20) $\forall x F x \rightarrow F y$

Supposons que dans le domaine de $\omega, D(\omega)$, tous les objets tombent sous l'extension du prédicat $« F »$. L'antécédent du conditionnel (20) est vrai dans une telle situation. Pour autant, on peut assigner à la variable $y$ pour valeur un objet qui n'est pas, lui, dans $\omega-$ on peut le faire, puisque nous avons stipulé qu'il était possible de faire référence à des objets absents du domaine de quantification d'un monde. Si l'on suppose que dans la situation que nous imaginons, cet objet appartient à l'extension du prédicat « $F »$, le conséquent du conditionnel est faux. En conséquence, (20) est falsifiée dans cette situation. Contrairement à ce que soutenait Frege, il semble donc difficile de maintenir la loi logique d'instanciation universelle - du moins si l'on adhère à la sémantique de Kripke.

Les modèles de Kripke sont souvent considérés comme apportant une réponse entièrement satisfaisante aux contre-exemples aux formules de Barcan. Ces formules sont falsifiées dans un tel cadre théorique, et les contre-exemples peuvent donc être acceptés, conformément à nos meilleurs jugements modaux. De nombreux philosophes y voient, du coup, une représentation de nos intuitions métaphysique bien plus satisfaisante que le cadre minimal impliquant les formules de Barcan. À vrai dire, il arrive que ce cadre sémantique soit aujourd'hui adopté, lors de discussions métaphysiques, sans guère de justification préalable 22 .

Le point le plus important pour notre propos réside en ce que l'adoption des domaines variables conduit naturellement à revenir sur l'analyse frégéenne, déflationniste, de l'existence. Comme le remarque Kripke, l'hypothèse des domaines variables permet de définir rigoureusement un prédicat d'existence « $E »$, exprimant une propriété du premier ordre : «l'existence est un prédicat monadique », affirme

22 Voir par exemple C. S. CHIHARA, The Worlds of Possibility : Modal Realism and the Semantics of Modal Logic, Clarendon Press, Oxford, 1998. 
$\mathrm{Kripke}^{23}$, et l'on peut définir son extension comme étant, dans chaque monde $\omega$, exactement identique au domaine du monde en question. Soit :

$\forall x(E x \leftrightarrow \exists y(x=y))$

La propriété d'exister peut alors être représentée, en utilisant les notations du lambdacalcul, ainsi :

$\lambda x \exists y(x=y)$

Dans le cadre kripkéen, « exister » se comporte véritablement comme un prédicat monadique. De même que le prédicat « animal », par exemple, possède des extensions variables dans différents mondes possibles - il n'est pas nécessaire que l'ensemble des animaux soit le même dans tous les mondes - , le prédicat « exister »s'applique dans des mondes différents à des objets différents. Le prédicat ne s'applique pas à l'objet Pégase dans le monde actuel ; mais il y a un monde possible dans lequel le prédicat s'applique correctement à cet objet : un monde où il existe au moins un cheval ailé nommé « Pégase ». L'existence devient un trait contingent de certains objets, qu'ils peuvent, dans un monde, instancier ou non. On voit donc que la théorie de Kripke contredit le second principe frégéen : exister, ce n'est pas être un objet possible de référence ; il y a des objets de référence qui se trouvent exister dans notre monde ; mais certains objets de référence n'y existent pas : ils existent dans d'autres mondes. Le second principe frégéen - le principe de l'existence des objets de référence - est abandonné, mais le premier - le principe de négation signifiante - semble bien satisfait par l'approche de Kripke : de même que tous les objets ne tombent pas sous le prédicat « animal » dans le monde réel, tous les objets ne tombent pas sous le prédicat « exister »-puisque Pégase, par exemple, ne tombe pas sous ce prédicat.

\section{4.}

Or il me semble que l'on aurait tort d'abandonner le second principe de Frege. Je voudrais montrer - à la suite de plusieurs auteurs ${ }^{24}$ - que l'hypothèse des domaines variables n'apporte qu'une solution très insatisfaisante aux contre-exemples aux

${ }^{23}$ KRIPKE, « Semantical considerations », cit., in L. LINSKY, (ed.), Reference and Modality, Oxford University Press, Oxford, 1971, 70.

24 Voir B. LinsKY B. / E. ZALTA, « In defense of the simplest quantified modal logic », cit., 431-458 ; M. J. CRESSWELL, «In defense of the Barcan formula », Logique et Analyse, 135-136, 271-282 ; et surtout T. WILLIAMSON, « Bare possibilia », cit., 257-273. 
formules de Barcan. Je proposerai ensuite une réinterprétation des formules de Barcan susceptible de les mettre à l'abri de ces contre-exemples.

Nous l'avons vu, les règles logiques associées au système sémantique de Kripke ne sont pas celles de la théorie classique de la quantification. En particulier, les règles d'instanciation universelle et de généralisation existentielle doivent être modifiées, $\mathrm{du}$ moins si l'on veut garder le principe de bivalence. Supposons en effet que toute formule est ou bien vraie, ou bien fausse, dans un monde possible donné. Supposons de plus que Socrate n'existe pas dans un monde $\omega$. Dans ce monde, la traduction de la phrase « Socrate est philosophe » est fausse, puisqu'elle ne peut pas être vraie. Il peut pourtant s'agir d'un monde où tous les objets sont philosophes. La conjonction suivante est donc vraie dans ce monde, ce qui constitue une transgression de la règle d'instanciation universelle :

(21) $\forall x P x \wedge \neg P s$

Dans une sémantique kripkéenne contenant des constantes d'individus, il n'est donc pas simplement possible, mais aussi nécessaire, d'introduire un prédicat d'existence de premier ordre. On voit à quel point ce type de sémantique s'écarte de l'orthodoxie frégéenne : l'existence n'est pas seulement une propriété d'individus ; c'est en outre une propriété qu'il faut nécessairement mentionner dans la formulation de certaines des lois logiques les plus fondamentales. La règle d'instanciation universelle doit en effet être retranscrite de la façon suivante :

$\forall x \phi x$

$$
E x \rightarrow \phi s
$$

Ce qu'on peut paraphraser ainsi :

Si tous les $x$ possèdent la propriété $\phi$, alors $s$ possède cette propriété, $d u$ moins s'il existe.

Le prédicat d'existence joue alors un rôle central dans la formulation des règles de la quantification, puisqu'il devient nécessaire de s'assurer que le référent d'une constante existe avant d'appliquer les règles d'instanciation universelle ou de généralisation existentielle. Le système de quantification qui en résulte se complique donc notablement, ce qui n'est en soi pas une bonne chose.

Il existe cependant un argument véritablement décisif contre la sémantique des domaines variables, qui a récemment été développé dans deux articles, de Linsky et 
Zalta d'une part, et de Williamson de l'autre ${ }^{25}$. Il repose sur un examen attentif de la formulation, dans le métalangage de la théorie sémantique, des conditions de vérité des formules modales. Pour qu'une analyse sémantique soit philosophiquement éclairante, il faut que l'on puisse l'appliquer aux expressions utilisées dans le métalangage de la théorie. Il est certain que si l'on utilise des expressions, dans le métalangage, en les dotant d'une sorte de signification qui n'est pas analysée dans le langage objet, l'analyse, quoique éventuellement utile d'un point de vue technique, n'apporte pas d'éclaircissement philosophique sur la nature des concepts utilisés dans le métalangage.

Considérons cependant attentivement la façon dont se trouvent analysés les contreexemples à BF que nous avons présentés, en précisant les formalisations des deux contre-exemple principaux ainsi que leurs conditions de vérité :

[a.] Pierre Martin aurait pu avoir un fils (vrai).

[b.] $\ \exists x F x a$.

[c.] Il existe un monde possible $\omega$ tel que pour au moins une assignation d'une valeur $b$ à la variable $x, b$ appartient au domaine de $\omega$, et $\langle b, a\rangle$ appartient à la dénotation de « $F$ » dans $\omega$.

[a.] Il existe une personne qui aurait pu être le fils de Pierre Martin (faux).

[b.] $\exists x \gg F x a$.

[c.] Il existe une assignation d'une valeur $c$ à la variable $x$ telle que $b$ appartient au domaine du monde réel, et telle qu'il existe un monde possible $\omega$ dans lequel $\langle c, a\rangle$ appartient à la dénotation de « $F$ »

On voit, dans cette analyse des conditions de vérité des formules modales, que les quantificateurs du métalangage ne sont pas restreints aux domaines des mondes possibles, contrairement aux quantificateurs du langage objet. C'est un peu comme si Frege avait raison dans le métalangage de la théorie censée lui donner tort : dans ce métalangage, tous les objets possibles peuvent être les valeurs de variables de quantification, alors que ce n'est pas le cas dans le langage objet. Reprenons à Thimothy Williamson la conclusion de cet argument : « ainsi, l'approche faisant appel à des domaines non modaux relativisés n'est pas satisfaisante philosophiquement : elle

\footnotetext{
25 Voir LinSKY / ZALTA, «In defense... », cit., et WiLLIAMSON, « Bare possibilia », cit.
} 
quantifie d'une façon que sa propre théorie de la quantification ne peut pas expliquer $»^{26}$.

De ce qui précède pourrait naître le soupçon selon lequel le prédicat d'existence introduit dans le cadre kripkéen ne satisfait qu'en apparence le principe de négation signifiante. Ce soupçon est justifié : ce n'est que lorsque le théoricien utilise, illicitement d'un point de vue métaphysique, le métalangage de sa théorie sémantique, que le prédicat satisfait cette contrainte frégéenne. Certes, il y a des mondes possibles dans lesquels se trouvent des objets qui n'existent pas dans le monde actuel, et le prédicat d'existence, tel que nous l'utilisons dans le monde actuel, ne s'applique pas à tous les objets. Le fait que le prédicat d'existence ne s'applique qu'à certains objets, nous l'apprenons lorsque nous comprenons que certains énoncés modaux sont justifiés : (22) Pégase n'existe pas, mais Pégase aurait pu exister.

(23) J'existe, mais j'aurais pu ne pas exister.

Soulignons bien que, si l'on prend l'analyse de Kripke au pied de la lettre, il n'y a rien dans le monde réel auquel on ne puisse pas appliquer la prédicat d'existence : « tout existe » est une vérité logique dans ce système, et la formule :

(24) $\forall x E x$

est vraie dans tous les modèles et tous les mondes possibles.

Mais si tout existe, comment pouvons-nous affirmer de façon justifiée, à propos de certains objets, qu'ils n'existent pas ? L'analyse sémantique des énoncés modaux nous donne une réponse : un objet qui n'existe pas, relativement au monde réel, est un objet qui existe, mais dans un monde simplement possible. C'est parce que nous pouvons faire référence à ces objets qui n'existent que relativement à d'autres mondes possibles que nous pouvons utiliser, de façon douée de sens, un prédicat d'existence. Autrement dit, nous pouvons utiliser le prédicat d'existence parce que nous savons qu'il y a des objets qui n'existent que dans des mondes simplement possibles. Mais en disant cela, on voit bien que nous sommes obligés de quantifier non seulement sur les objets qui existent dans le monde réel, mais aussi sur les objets qui existent dans d'autres mondes possibles. Nous sommes donc obligés de reconnaître qu'il y a des objets qui n'existent pas, contrairement à ce qu'implique le système de Kripke.

Le fait que la théorie de Kripke utilise les quantificateurs de façon différente lorsqu'il s'agit du langage objet et lorsqu'il s'agit du métalangage apparaît ici de façon

26 WILLIAMSON, « Bare possibilia », cit., 263. 
nette. Pour donner un sens au prédicat d'existence, nous devons reconnaître qu'il y a des objets, dans d'autres mondes possibles, qui n'existent pas dans notre monde (principe de négation signifiante); mais lorsque nous faisons cela, nous quantifions bien sur de simples possibles, et pas simplement sur les objets qui existent dans notre monde ; ce qui n'est pas supposé faisable si l'analyse kripkéenne de la quantification modale est correcte.

5.

En raison de toutes ces difficultés, je propose de réinterpréter les contre-exemples aux formules de Barcan, afin de montrer que la thèse d'un unique domaine de quantification peut être maintenue. L'idée centrale de cette réinterprétation est la suivante : adopter un unique domaine de quantification implique que nous reconnaissions l'existence actuelle de tous les objets de ce domaine, mais pas que nous reconnaissions leur existence au sens spatio-temporel du terme. Les contre-exemples aux formules de Barcan perdent en effet toute leur force dès lors que nous admettons l'existence des possibilia. Considérons de nouveau le couple de formules censé falsifier BF :

(25) Pierre Martin aurait pu avoir un fils.

(26) Il existe un objet tel qu'il aurait pu être le fils de Pierre Martin.

Pourquoi jugeons-nous que (26) est fausse ? Parce que nous donnons, me semble-t-il, une signification trop restrictive au verbe « exister ». Si nous considérons qu'existent uniquement dans le monde des objets spatio-temporels, alors l'énoncé (26) apparaîtra certainement vrai. Mais il semble que nous puissions donner un autre sens à la quantification existentielle : selon Frege, suivi en cela par Quine, elle reflète nos engagements ontologiques, quels que soient les types d'objets vis-à-vis desquels nous sommes engagés. Nous pouvons reconnaître l'existence d'une entité, sans pour autant être capables de la localiser dans l'espace et dans le temps : nos engagements portent sur ce qu'il y a en général, et pas uniquement sur ce qu'il y a dans l'espace et dans le temps.

Il me semble que c'est exactement ainsi qu'il faut interpréter (26) : la formule est vraie, et elle est bien justifiée dans le système le plus satisfaisant de logique modale, mais elle n'implique pas que l'existence affirmée doive être comprise au sens spatio- 
temporel. C'est parce que nous avons tendance à interpréter ainsi le concept d'existence que certaines intuitions nous poussent à rejeter, à tort, (26). A condition de comprendre l'expression « il existe » dans son sens le plus large, la vérité de (26) peut tout à fait être soutenue : il y a bien, après tout, une entité dont on peut dire qu'elle aurait pu être le fils de Pierre Martin, même si cette entité ne peut être localisée dans l'espace-temps. Si l'on donne une interprétation aussi générale à l'expression « il y a », et donc au quatificateur existentiel «il existe $x$ tel que... », les formules de Barcan apparaissent clairement comme des vérités logiques. Considérons de nouveau la formulation la plus intuitive de BF :

S'il avait pu être le cas qu'il y ait une chose possédant la propriété $\phi$, alors il y a une chose dont il aurait pu être le cas qu'elle ait la propriété $\phi$.

Si l'on suppose que « il y a » ne signifie pas « il y a dans l'espace temps actuel », la formule devient trivialement vraie. Dire « il aurait pu être le cas qu'il y ait une chose (qui n'existe éventuellement pas dans l'espace et le temps, mais qui aurait pu y exister) possédant la propriété $\phi$ » revient évidemment exactement à dire « il y a une chose (qui n'existe éventuellement pas dans l'espace et le temps, mais qui aurait pu y exister) dont il aurait pu être le cas qu'elle ait la propriété $\phi »$.

Je rejoins donc l'interprétation dite « possibiliste » des formules de Barcan. Il est possible, dans le cadre de cette approche, d'introduire un prédicat d'existence ayant précisément un sens spatio-temporel. Le prédicat d'existence possède alors une signification restreinte - n'existent que les objets qui sont dans l'espace et dans le temps - et le quantificateur existentiel un sens plus général. Ce prédicat ne pourra cependant pas être défini uniquement à l'aide de symboles logiques, puisque tous les objets du domaine de quantification n'existent pas, au sens spatio-temporel. Ainsi, le nombre 3 n'existe pas en ce sens, pas plus que le fils simplement possible de Pierre Martin. La seule façon raisonnable d'introduire un tel prédicat me semble être de le caractériser comme exprimant une relation entre les individus du domaine de quantification $D$ et les structures spatio-temporelles associées à chaque monde possible. Soit $S T_{\omega}$ la structure d'espace-temps associée au monde possible $\omega$. Nous définirons ainsi l'extension du prédicat $E$ relativement à chaque monde possible :

Pour tout objet du domaine de quantification $x, x$ existe dans le monde $\omega$ si et seulement si $x$ appartient à $S T_{\omega}$. 
Le point philosophiquement essentiel est que le prédicat d'existence ainsi défini n'est pas un prédicat logique ; il n'exprime pas une propriété intrinsèque des objets de quantification, mais simplement une relation : un objet existe à condition d'entretenir une relation appropriée avec l'espace-temps d'un monde possible ; il n'existe pas, dans ce monde possible, dans le cas contraire.

Pour que notre approche soit plausible, il faut également supposer que la quantification est en général, dans la langue naturelle, restreinte par le prédicat d'existence. Considérons par exemple une loi de la nature, représentée par une formule générale du premier ordre :

(27) $\forall x(F x \rightarrow G x)$.

Je soutiens que la forme logique correcte de cette formule est en fait la suivante : (28) $\forall x((E x \wedge F x) \rightarrow G x)$.

Autrement dit, les lois de la nature ne portent pas sur tous les objets qu'il y a, mais uniquement sur les objets qu'il y a dans l'espace-temps, ou encore, sur tous les objets qui existent (au sens du prédicat relationnel d'existence que nous venons de caractériser). Une telle hypothèse sémantique paraît plausible, puisque les domaines de quantification sont toujours restreints dans la langue naturelle 27.

Soulignons que la loi de généralisation existentielle peut s'appliquer librement dans ce nouveau cadre, même si l'on admet qu'il soit possible de nommer les possibilia. En effet, (29) s'analyse de la façon, non paradoxale, précisée en (30) :

(29) Pégase n'existe pas, mais il aurait pu exister.

(30) Pégase n'appartient pas à la structure d'espace-temps associée au monde actuel, mais il existe un monde possible possédant une structure d'espace-temps qui contient Pégase.

Du coup, on peut généraliser existentiellement sans paradoxe à partir de (29) : « Pégase n'existe pas » implique logiquement «Il y a un objet qui n'existe pas »; et « Pégase n'appartient pas à la stucture d'espace-temps associée au monde réel » implique logiquement « Il y a au moins une chose qui n’appartient pas à la structure d'espace-temps associée au monde réel ». La généralisation n'est pas paradoxale : il y a des objets qui n'existent pas dans la structure spatio-temporelle de tel monde, de même

27 Voir F. RÉCANATI, « Domains of discourse », Linguistics and Philosophy, 19 (1996), $445-475$. 
qu'il y a des objets qui existent uniquement avant telle date, ou après telle date, ou dans tel ou tel lieu et pas dans tel autre lieu.

6.

Contrairement à ce que Frege lui-même pensait, sans doute en raison de son peu d'intérêt pour les modalités, la thèse centrale selon laquelle exister n'est rien d'autre, au sens fort, qu'appartenir à un domaine de quantification, ne peut être défendue qu'à condition d'introduire un prédicat d'existence. La thèse sémantique de Frege apparaît donc fausse : la plupart des énoncés d'existence attribuent bien des propriétés à des individus. Le prédicat d'existence, néanmoins, n'admet de définition que relationnelle. Et c'est me semble-t-il le point important en ce qui concerne la position frégéenne : il n'exprime pas une propriété intrinsèque des individus, et ne peut pas être défini à l'aide du seul vocabulaire logique. Métaphysiquement, l'existence n'est donc pas une propriété intrinsèque des individus : quoique sa thèse sémantique soit fausse, la thèse métaphysique de Frege semble bien fondée. 